OPEN ACCESS

Edited by:

Sinuhe Hahn,

University of Basel, Switzerland

Reviewed by:

Peter Sedlmayr,

Medical University of Graz, Austria

Tanja Premru-Sršen,

University Medical Center

Ljubljana, Slovenia

*Correspondence:

Dilly O. C. Anumba

d.o.c.anumba@sheffield.ac.uk

Specialty section:

This article was submitted to

Obstetrics and Gynecology,

a section of the journal

Frontiers in Medicine

Received: 18 August 2016 Accepted: 28 September 2016

Published: 10 October 2016

Citation:

Amabebe E, Reynolds S, Stern V,

Stafford G, Paley M and Anumba DOC (2016) Cervicovaginal Fluid Acetate: A Metabolite Marker of

Preterm Birth in Symptomatic

Pregnant Women.

Front. Med. 3:48.

doi: 10.3389/fmed.2016.00048

\section{Cervicovaginal Fluid Acetate: A Metabolite Marker of Preterm Birth in Symptomatic Pregnant Women}

\author{
Emmanuel Amabebe ${ }^{1}$, Steven Reynolds ${ }^{2}$, Victoria Stern ${ }^{1}$, Graham Stafford ${ }^{3}$, Martyn Paley $^{2}$ \\ and Dilly O. C. Anumba ${ }^{1 *}$ \\ 'Academic Unit of Reproductive and Developmental Medicine, University of Sheffield, Sheffield, UK, ${ }^{2}$ Academic Unit of Radiology, \\ University of Sheffield, Sheffield, UK, ${ }^{3}$ Integrated BioSciences, School of Clinical Dentistry, University of Sheffield, Sheffield, UK
}

Changes in vaginal microbiota that is associated with preterm birth (PTB) leave specific metabolite fingerprints that can be detected in the cervicovaginal fluid (CVF) using metabolomics techniques. In this study, we characterize and validate the CVF metabolite profile of pregnant women presenting with symptoms of threatened preterm labor (PTL) by both ${ }^{1} \mathrm{H}$-nuclear magnetic resonance spectroscopy (NMR) and enzyme-based spectrophotometry. We also determine their predictive capacity for PTB, singly, and in combination, with current clinical screening tools - cervicovaginal fetal fibronectin (FFN) and ultrasound cervical length (CL). CVF was obtained by high-vaginal swabs from 82 pregnant women with intact fetal membranes presenting between 24 and 36 weeks gestation with symptoms of threatened, but not established, PTL. Dissolved CVF samples were scanned with a $400 \mathrm{MHz}$ NMR spectrometer. Acetate and other metabolites were identified in the NMR spectrum, integrated for peak area, and normalized to the total spectrum integral. To confirm and validate our observations, acetate concentrations (AceConc) were also determined from a randomly-selected subset of the same samples $(n=57)$, by spectrophotometric absorption of NADH using an acetic acid assay kit. CVF FFN level, transvaginal ultrasound $\mathrm{CL}$, and vaginal $\mathrm{pH}$ were also ascertained. Acetate normalized integral and AceConc were significantly higher in the women who delivered preterm compared to their term counterparts $(P=0.002$ and $P=0.006$, respectively). The ${ }^{1} \mathrm{H}$-NMR-derived acetate integrals were strongly correlated with the AceConc estimated by spectrophotometry $(r=0.69 ; P<0.0001)$. Both methods were equally predictive of PTB <37 weeks (acetate integral: $\mathrm{AUC}=0.75,95 \% \mathrm{Cl}=0.60-0.91$; AceConc: $A \cup C=0.74,95 \% \mathrm{Cl}=0.57-0.90$, optimal predictive cutoff of $>0.53 \mathrm{~g} / \mathrm{l})$, and of delivery within 2 weeks of the index assessment (acetate integral: $\mathrm{AUC}=0.77,95 \% \mathrm{Cl}=0.58$ 0.96; AceConc: $\mathrm{AUC}=0.68,95 \% \mathrm{Cl}=0.5-0.9$ ). The predictive accuracy of CVF acetate was similar to $C L$ and FFN. The combination of CVF acetate, FFN, and ultrasound CL in a binary logistic regression model improved the prediction of PTB compared to the three markers individually, but CVF acetate offered no predictive improvement over ultrasound CL combined with CVF FFN. Elevated CVF acetate in women with symptoms of PTL appears predictive of preterm delivery, as well as delivery within 2 weeks of presentation. An assay of acetate in CVF may prove of clinical utility for predicting PTB.

Keywords: cervicovaginal fluid, microbiota, metabolite, acetate, preterm birth 


\section{INTRODUCTION}

Approximately 15 million babies are born prematurely annually. Preterm birth (PTB, birth before 37 weeks of gestation), is a global problem costing health care resources in excess of \$26 billion in the USA and $£ 3$ billion in the UK annually. It is the commonest cause of infant morbidity and mortality worldwide. About $35 \%$ of the world's 3.1 million neonatal deaths annually are due to complications of PTB. Many surviving preterm babies face a lifetime of disability including cerebral palsy, learning, visual, and respiratory disorders amongst others (1-3). Preventing prematurity remains limited by poor prediction, and current tests to identify women at risk are inadequate.

Although the etiology of PTB is multifactorial, infection and inflammation are associated with most spontaneous preterm deliveries (3). However, the etiological mechanisms and pathogenesis of these observations are unclear. Changes in the vaginal microecology of commensal and potentially pathogenic organisms such as bacterial vaginosis (BV) - a polymicrobial vaginal infection common in reproductive-aged women - may influence the initiation of PTB (3). BV is characterized by a shift (dysbiosis) from the healthy Lactobacillus-dominated vaginal microflora to an abnormal microbial environment dominated by mixed anaerobes e.g., Gardnerella, Fusobacterium, Bacteroides, Prevotella, Mobiluncus, Mycoplasmas, etc. These anaerobes synthesize metabolic acids such as acetate, butyrate, propionate, and succinate, thereby increasing the vaginal $\mathrm{pH}$ and inhibiting chemotaxis of immunocompetent cells (4-6). This, in turn, enhances the proliferation of these infectious organisms, leading to ascending intrauterine infection and the establishment of an NF- $\kappa B$-coordinated inflammatory state (6) that recruits proinflammatory cytokines (e.g., IL- $1 \beta$, IL-4, IL-6, IL-17, TNF- $\alpha$, and INF- $\gamma$ ) and chemokines (e.g., IL- 8 and RANTES), stimulating the production of matrix metalloproteinases (MMPs) and arachidonic acid metabolites such as prostaglandins (PGs) and hydroxyeicosatetraenoic acids (HETE). The MMPs degrade and digest the cervical extracellular matrix and fetal membranes, while the arachidonic acid metabolites modulate myometrial contractility leading to cervical remodeling, preterm labor (PTL), premature membrane rupture, and ultimately PTB (3, 7-10).

Determination of vaginal microbiota metabolite signatures has provided clinically useful insight into the pathophysiology of ascending genital tract infection and subsequent reproductive outcome (11-13). Metabolic acids (e.g., acetate and succinate) produced in large amounts by female genital microbiota (dominated by mixed anaerobic bacteria and deficient in the protective lactic acid-producing Lactobacillus species) have been shown to exhibit deleterious immunomodulatory functions (6). These include increasing the $\mathrm{pH}$ of the vaginal ecosystem, inducing the production of pro-inflammatory cytokines, and paralyzing the chemotaxis of neutrophils, monocytes, and other immunocompetent cells $(4,5,14)$. These encourage luxuriant growth and proliferation of potentially pathogenic bacteria, ascending genital infection, microbial invasion of the amniotic cavity, and inflammation of the fetal membranes and other gestational tissues. The cumulative effect of this sequence of events during gestation is the release of PGs and MMPs, and consequently cervical remodeling, myometrial contraction, preterm premature rupture of membranes, PTL, and preterm delivery (3, 8, 10, 15-17).

Interestingly, the changes in the vaginal microbiota also leave specific signature metabolite fingerprints that can be detected in the cervicovaginal fluid (CVF) using metabolomics techniques such as ${ }^{1} \mathrm{H}$-nuclear magnetic resonance spectroscopy (NMR) $(11,13)$. In addition to enhancing our understanding of the pathogenesis of inflammation-induced PTB, it is plausible that CVF metabolite profiling can enable the development of clinical predictive tests for identifying women at risk of PTB. Besides, some of these metabolites have been useful in the diagnosis of BV (12), and more recently, we have demonstrated the predictive potential of CVF acetate (derived by ${ }^{1} \mathrm{H}-\mathrm{NMR}$ ) for PTB especially in symptomatic pregnant women (18). Hence, in this study employing commercial spectrophotometric assay techniques with potential for clinical applicability, we validated metabolite profiles previously determined by ${ }^{1} \mathrm{H}-\mathrm{NMR}$ spectroscopy in CVF obtained from a large cohort of pregnant women presenting with symptoms suggestive of threatened PTL and determined their prognostic capacity for PTB singly and in conjunction with widely employed clinical assessment methods such as quantitative fetal fibronectin (FFN) and ultrasound cervical length (CL). We hypothesized that, compared to their term-delivered counterparts; symptomatic pregnant women who ultimately delivered prematurely would have significantly different CVF metabolite profiles with predictive accuracies comparable to current clinical tests such as cervicovaginal FFN and ultrasound-derived CL.

\section{MATERIALS AND METHODS}

\section{Study Cohort and Sampling}

Cervicovaginal fluid was obtained by high-vaginal swabs (Deltalab Eurotubo 300263, Fisher Scientific, UK) from pregnant women presenting to the Triage Delivery Suites of the Jessop Wing Hospital, Sheffield, UK, with symptoms suggestive of threatened, but not established, PTL (i.e., regular uterine contractions at least once every $10 \mathrm{~min}$ and cervical dilatation $<3 \mathrm{~cm}$ ), between 24 and 36 weeks gestation $(n=82)$. Women with multiple gestation, symptoms or signs of genitourinary infection, and history of abnormal cervical cytology in the last 3 years, ruptured fetal membranes, and prior vaginal examinations at presentation were excluded from the study. Study participants were recruited between January 2014 and September 2015 and closely monitored until delivery outcome was ascertained.

Vaginal swabs were obtained from participants prior to any vaginal examination or clinical treatment intervention such as therapy with antibiotics, steroids, tocolytics, or any vaginal pessary. The obtained CVF samples were instantly processed or stored at $-20^{\circ} \mathrm{C}$ in preparation for metabolite analyses. At presentation, clinical research staff also ascertained CVF FFN, ultrasound $\mathrm{CL}$, and vaginal $\mathrm{pH}$. The clinical course and delivery outcomes of participants were subsequently ascertained.

These studies were approved by the Yorkshire \& Humber (Sheffield) Committee of the UK National Research Ethics Service (REC Number 13/YH/0167). 


\section{'H-NMR Spectroscopy}

Cervicovaginal fluid samples were prepared and analyzed by ${ }^{1} \mathrm{H}-\mathrm{NMR}$ as previously described (18). Dacron swab saturated with CVF obtained from the posterior fornix of the vagina was immediately returned to the sterile transport tube, labeled correctly, and stored briefly at $-20^{\circ} \mathrm{C}$ pending extraction into solution. A physiological extraction solution of $600 \mu$ l Phosphate Buffered Saline at $\mathrm{pH} 7.4$ was added to the cut end of the swab saturated with CVF sample in a clean $1.5 \mathrm{ml}$ microfuge tube and vortexed for $5 \mathrm{~min}$. The swab was safely discarded after CVF had been washed off into solution. Following centrifugation $(13,000 \mathrm{rpm} \times 3 \mathrm{~min})$, the supernatant was separated and carefully aspirated into a different clean $1.5 \mathrm{ml}$ microfuge tube and stored at $-80^{\circ} \mathrm{C}$ ready for analysis. Four hundred microliters of each NMR sample comprising of $380 \mu \mathrm{l}$ of CVF in solution and $20 \mu \mathrm{l}$ of deuterium oxide $\left(\mathrm{D}_{2} \mathrm{O}\right)$ was transferred into a $5 \mathrm{~mm} \mathrm{NMR}$ sample tube (Norell, Marion, NC, USA), ready for scanning.

${ }^{1} \mathrm{H}-\mathrm{NMR}$ spectra of CVF samples were acquired at approximately $294 \mathrm{~K}$ with a $9.4 \mathrm{~T}$ (400 MHz) Bruker Avance III MR spectrometer (Bruker BioSpin GmbH, Karlsruhe, Germany), with $5 \mathrm{~mm}$ broadband observe probe using a Watergate water suppression pulse sequence (number of scans, NS $=256$, relaxation time, $\mathrm{D} 1=5 \mathrm{~s}$, acquisition time, $\mathrm{AQ}=1 \mathrm{~s}$, sweep width, $\mathrm{SW}=20.6 \mathrm{ppm}$, time domain, $\mathrm{TD}=16446) .{ }^{1} \mathrm{H}-\mathrm{NMR}$ spectra phase and baseline correction as well as data processing was performed using the Bruker TOPSPIN 2.1.6 software.
In order to confirm the structure of the identified metabolites and assign them to the ${ }^{1} \mathrm{H}-\mathrm{NMR}$ spectral peaks (Figure 1), the following 2-D NMR spectra were acquired ${ }^{1} \mathrm{H}-{ }^{13} \mathrm{C}$ presatheteronuclear single quantum correlation spectroscopy (HSQC) $\mathrm{NS}=1024, \mathrm{D} 1=1 \mathrm{~s}, \mathrm{AQ}=0.078 \times 0.006 \mathrm{~s}, \mathrm{SW}=10.0 \times 150 \mathrm{ppm}$, $\mathrm{TD}=624 \times 180 ;{ }^{1} \mathrm{H}-{ }^{13} \mathrm{C}$ presat-heteronuclear multiple bond correlation spectroscopy $(\mathrm{HMBC})-\mathrm{NS}=1024, \mathrm{D} 1=1 \mathrm{~s}$, $\mathrm{AQ}=0.128 \times 0.005 \mathrm{~s}, \mathrm{SW}=10.0 \times 230 \mathrm{ppm}, \mathrm{TD}=1024 \times 200$; ${ }^{1} \mathrm{H}-{ }^{1} \mathrm{H}$ watergate-double quantum filtered correlation spectroscopy $(\mathrm{DQFCOSY})-\mathrm{NS}=256, \mathrm{D} 1=0.5 \mathrm{~s}, \mathrm{AQ}=0.832 \times 0.022 \mathrm{~s}$, $\mathrm{SW}=9.0 \times 9.0 \mathrm{ppm}, \mathrm{TD}=6000 \times 160$; and ${ }^{1} \mathrm{H}-{ }^{1} \mathrm{H}$ presat-clean total correlation spectroscopy (TOCSY) $-\mathrm{NS}=16, \mathrm{D} 1=1.5 \mathrm{~s}$, $\mathrm{AQ}=0.284 \times 0.071 \mathrm{~s}, \mathrm{SW}=9.0 \times 9.0 \mathrm{ppm}, \mathrm{TD}=2048 \times 512$. A representative 2-D ${ }^{1} \mathrm{H}-{ }^{13} \mathrm{C}$ HSQC spectrum is shown in Figure S1 in Supplementary Material. All spectral peaks were referenced to the ${ }^{1} \mathrm{H}$ lactate signal at $\delta=1.30 \mathrm{ppm}$. The metabolite peak intensities were then assigned by matching their chemical shifts and multiplicity with previous publications $(13,19)$, SDBS Spectral Database for Organic Compounds (http://sdbs.db.aist. go.jp/sdbs/cgi-bin/cre_index.cgi), and Chenomx NMR Suite software package (Chenomx Inc., CA, USA, version 7.7). With the same experimental procedures, a sterile (unused) polystyrene Dacron swab was prepared and analyzed as a background control signal.

The identified ${ }^{1} \mathrm{H}$-NMR metabolite signals were integrated for peak area (which is proportional to metabolite concentration)

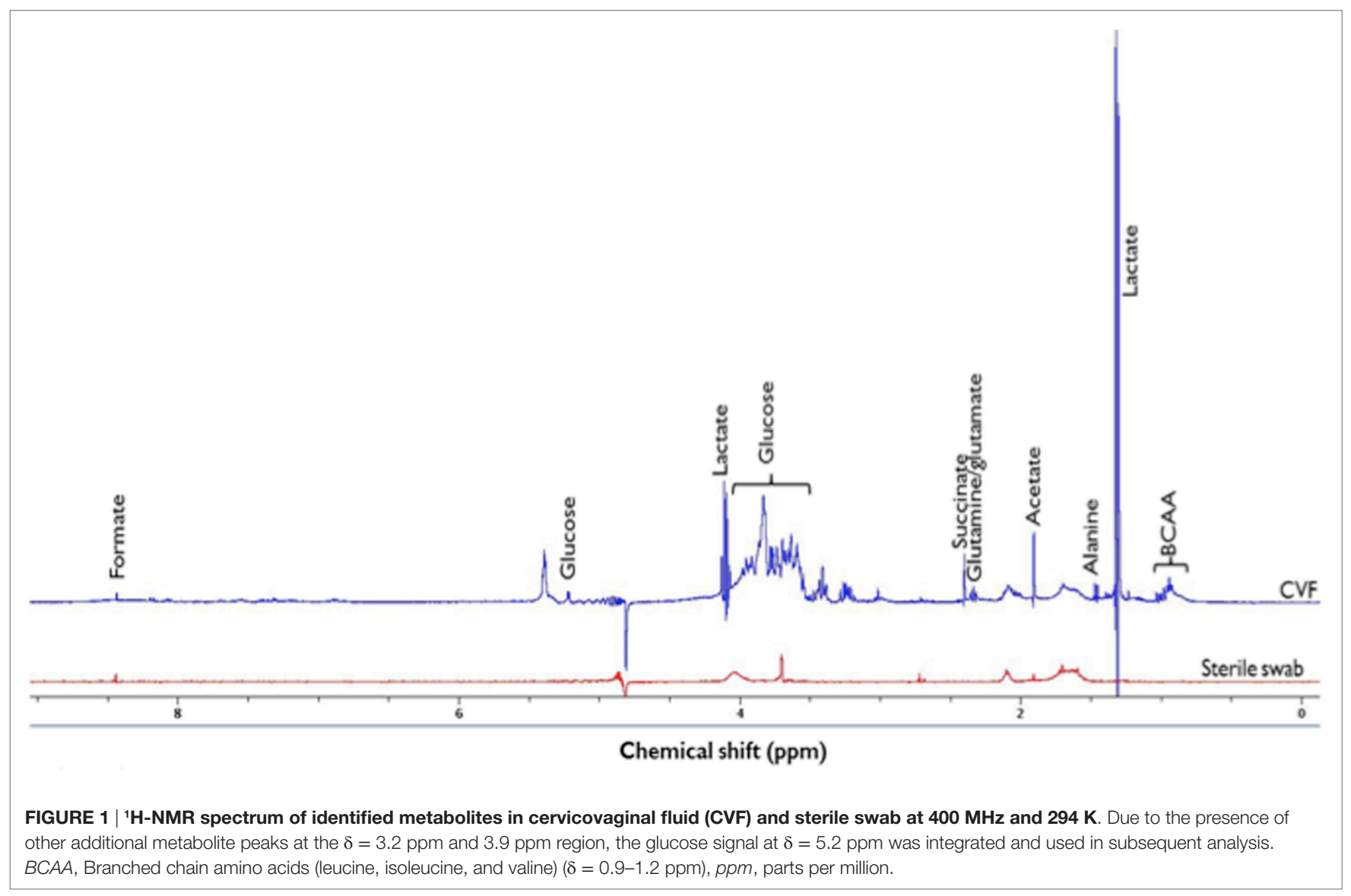


and normalized by dividing each integral by the total spectrum integral $(\delta=0.0-10.0 \mathrm{ppm}$, excluding the residual water signal $\delta=4.7-5.0 \mathrm{ppm}$ ) to provide a normalized integral (N.I.). This accounted for any differences in CVF concentration or variation in swab sampling.

\section{CVF Acetate Concentration Measurement}

To validate and confirm the data from NMR-derived acetate normalized integrals, predictive accuracy of CVF acetate for PTB and explore potential clinical translation, we determined absolute acetate concentration from a randomly-selected subset of CVF samples $(n=57)$ from the total cohort of participants, by spectrophotometric absorption of $\mathrm{NADH}$ using an assay kit for acetic acid (ADP-Glucokinase format, K-ACETGK 08/14, Megazyme, IE). The reaction is a positive reaction indicated by the increase in absorbance as demonstrated by the calibration curve $\left(R^{2}=0.975\right.$, detection limit: $1.8 \mathrm{~g} / \mathrm{l}$ ), with its lowest sensitivity limit $\sim 0.1 \mathrm{~g} / \mathrm{l}$ $\left(R^{2}=0.9997\right)$ (Figure S2 in Supplementary Material). The acetic acid GK assay kit is an endpoint type assay specific for acetate. All reagents were prepared, mixed, and stored according to the manufacturer's instruction. A single reaction mixture containing $3 \mu \mathrm{l} \mathrm{CVF} \mathrm{sample} \mathrm{in} \mathrm{PBS,} 200 \mu \mathrm{l}$ of reagent 1 (distilled water, buffer, and AK/PTA/ADP-GK/G6P-DH), and $20 \mu \mathrm{l}$ of reagent $2(\mathrm{NAD}+/ \mathrm{ATP} / \mathrm{D}$-glucose/CoA/PVP), was assayed for each sample. The reaction time was $\sim 5 \mathrm{~min}$ at $37^{\circ} \mathrm{C}$ after which the absorbance of the end product $\mathrm{NADH}$ was read at $340 \mathrm{~nm}$. All assays were performed using optical grade 96-well plates on a computer-controlled Infinite M200 microplate reader (TECAN, Chapel Hill) \{wavelength range $=230-1000 \mathrm{~nm}$; accuracy: $0-2$ OD: $\leq \pm[1 \%+10 \mathrm{mOD} ; 2-3 \mathrm{OD}: \leq \pm 2.5 \%$; Precision $<0.2 \%$ at $260 \mathrm{~nm}$; Linearity: $R^{2}=0.999(0-2$ OD)]\} (see Supplementary Material for more details).

\section{CVF Fetal Fibronectin, Ultrasound $\mathrm{CL}$, and Vaginal $\mathrm{pH}$}

Quantitative CVF FFN levels were determined using the 10Q Rapid FFN analyser (Hologic, MA, USA), vaginal pH using narrow range universal indicator paper ( $\mathrm{pH}$-Fix, Machery-Nagel, DE, USA), and CL by transvaginal ultrasonography.

\section{Data Analysis}

All statistical analyses, including receiver operating characteristics (ROC) curve determination were performed using MATLAB (Mathworks, Natick, MA, USA). The Wilcoxon rank-sum test was performed to compare differences in metabolite N.I. and concentration between term and preterm-delivered women within the group. Post hoc adjustment for multiple comparisons was undertaken by the Bonferroni test. Values are presented as mean \pm SEM (except where otherwise stated). The relationships between maternal clinical data, acetate concentration, and ${ }^{1} \mathrm{H}-$ NMR metabolite N.I. were determined by Pearson's correlation coefficients and $P$ values $<0.05$ were considered statistically significant. The predictive capacity of the CVF metabolites for PTB was determined by ROC curves for the following comparisons:

- Preterm (<37 weeks) vs. term births,

- Preterm birth $<32$ vs. $>32$ weeks gestation,

- $<2$ vs. $>2$ weeks from presentation to delivery.
A cutoff value of acetate concentration for predicting PTB $<37$ weeks gestation was also calculated from the ROC curve. The combined predictive accuracy of FFN, ultrasound CL, and CVF acetate was estimated by binary logistic regression and combined area under the ROC curve analysis (MedCalc Software bvba, BE, USA).

\section{RESULTS}

\section{Participants' Clinical Details and Pregnancy Outcome}

Participants' clinical characteristics and delivery outcomes are summarized in Table 1. Also, 18.3\% (15/82) of the women delivered preterm ( $<37$ weeks), with mean duration of time between presentation and delivery of $15.7 \pm 3.5$ (preterm) and $60.3 \pm 3.2$ days (term). Of the 15 PTBs recorded, 8 women ( $9.8 \%$ of the total study population) delivered before 32 weeks of gestation.

\section{${ }^{1} \mathrm{H}-$ NMR Derived Normalized Integrals}

A representative ${ }^{1} \mathrm{H}-\mathrm{NMR}$ spectrum of identified metabolites is shown in Figure 1.

Comparison of CVF metabolite N.I.s derived by ${ }^{1} \mathrm{H}-\mathrm{NMR}$ showed significantly higher $(0.03 \pm 0.01$ vs. $0.01 \pm 0.003$ a.u.; $P=0.002)$ acetate N.I. in women who delivered preterm compared to their term counterparts. There was about threefold increase in acetate N.I. in women who ultimately delivered preterm (Figure 2A).

TABLE 1 | Maternal clinical and demographic characteristics.

\begin{tabular}{|c|c|c|}
\hline \multirow[t]{2}{*}{ Characteristics } & \multicolumn{2}{|c|}{ Symptomatic pregnant women $24-36$ weeks gestation } \\
\hline & Preterm $(N=15)$ & Term $(N=67)$ \\
\hline Age (years) & $31.1 \pm 2.0^{\mathrm{b}}(22-48, n=15)$ & $26.6 \pm 0.7(16-44, n=67)$ \\
\hline $\mathrm{BMI}\left(\mathrm{kg} \mathrm{m}^{-2}\right)$ & $28.9+1.9^{a}(20.1-42.5, n=11)$ & $\begin{array}{c}25.1 \pm 0.6^{a}(17.4-41.6 \\
n=56)\end{array}$ \\
\hline $\begin{array}{l}\text { Previous history } \\
\text { of PTB (n) }\end{array}$ & 5 & 10 \\
\hline $\begin{array}{l}\text { Cigarette } \\
\text { smokers, } n(\%)\end{array}$ & $1(7)$ & $10(15)$ \\
\hline $\begin{array}{l}\text { Cervical length } \\
(\mathrm{mm})\end{array}$ & $21.1 \pm 4.8^{\mathrm{a}}(7-45, n=8)$ & $30.8 \pm 1.6^{\mathrm{ba}}(11-54, n=37)$ \\
\hline $\begin{array}{l}\text { Fetal fibronectin } \\
\text { conc. }(\mathrm{ng} / \mathrm{ml})\end{array}$ & $188 \pm 85^{\text {ba }}(5-501, n=6)$ & $15.2 \pm 3^{a}(1-74, n=37)$ \\
\hline $\begin{array}{l}\text { Gestational age } \\
\text { at presentation } \\
\text { (days) }\end{array}$ & $206 \pm 5.4(177-237, n=15)$ & $213 \pm 3.1(139-254, n=67)$ \\
\hline $\begin{array}{l}\text { Gestational age } \\
\text { at delivery (days) }\end{array}$ & $221 \pm 5.7(187-257, n=15)$ & $275 \pm 1.1(260-295, n=61)$ \\
\hline Vagina $\mathrm{pH}$ & $4.4 \pm 0.3^{\mathrm{a}}(3.6-6.1, n=7)$ & $4.2 \pm 0.1^{\text {a }}(3.6-6.1, n=36)$ \\
\hline $\begin{array}{l}\text { Prevalence of } \\
\text { PTB, \% }\end{array}$ & & \\
\hline
\end{tabular}

Data are presented as mean \pm SEM (range, $n$ ); BMI, body mass index; PTB, preterm birth; $N$, total number of term- or preterm-delivered women; $n$, actual number of women for each clinical parameter obtained.

aReduced study population (n) due to absence of participants' consent and/or data. ${ }^{b}$ Differences between preterm and term-delivered women, $P<0.05$. 
A

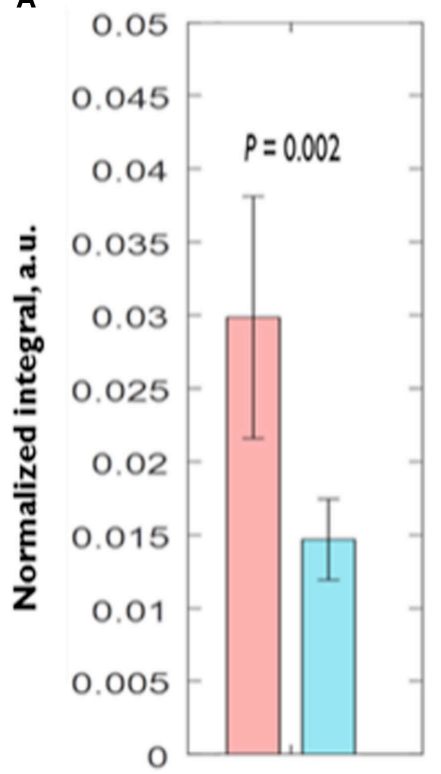

B

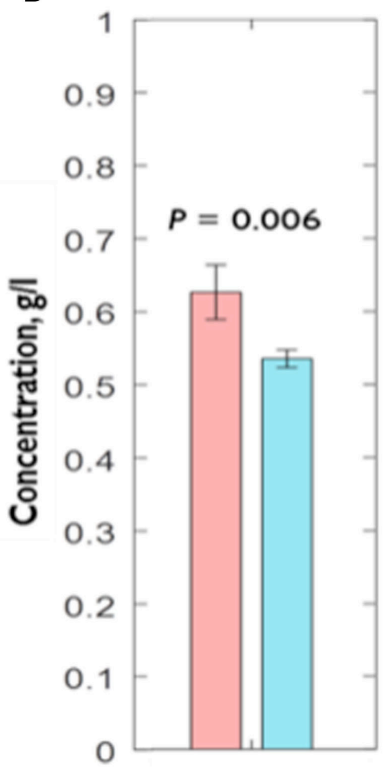

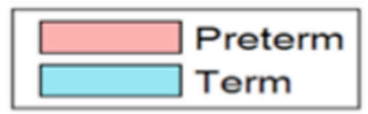

FIGURE 2 | Comparison of CVF (A) acetate normalized integral measured by ${ }^{1} \mathrm{H}-\mathrm{NMR}$ (B) acetate concentration measured by spectrophotometry between preterm and term women. Error bars = SEM; a.u., arbitrary unit.

TABLE 2 | Predictive accuracy of CVF acetate ${ }^{1} \mathrm{H}-\mathrm{NMR}$ normalized integral and concentration for preterm birth.

\begin{tabular}{|c|c|c|c|c|c|c|c|c|c|}
\hline & AUC & $95 \% \mathrm{Cl}$ & Sens (\%) & Spec (\%) & PPV (\%) & NPV (\%) & LR+ & LR- & $P$ value \\
\hline \multicolumn{10}{|c|}{ Delivery $<37$ weeks gestation } \\
\hline Acetate N.I. & 0.75 & $0.60-0.91$ & 60 & 85 & 47 & 91 & 4.0 & 0.2 & 0.001 \\
\hline AceConc & 0.74 & $0.57-0.90$ & 71 & 71 & 36 & 92 & 2.5 & 0.4 & 0.002 \\
\hline \multicolumn{10}{|c|}{ Delivery $<32$ weeks gestation } \\
\hline Acetate N.I. & 0.73 & $0.53-0.94$ & 88 & 59 & 21 & 97 & 2.1 & 0.5 & 0.01 \\
\hline AceConc & 0.63 & $0.40-0.87$ & 72 & 63 & 19 & 95 & 2.0 & 0.5 & 0.13 \\
\hline \multicolumn{10}{|c|}{ Delivery within 2 weeks of assay } \\
\hline Acetate N.I. & 0.77 & $0.58-0.96$ & 100 & 49 & 21 & 100 & 2.0 & 0.5 & 0.003 \\
\hline AceConc & 0.68 & $0.47-0.89$ & 67 & 79 & 29 & 95 & 3.1 & 0.3 & 0.045 \\
\hline
\end{tabular}

Acetate N.I., 'H-NMR acetate normalized integral; AceConc, acetate concentration measured by spectrophotometric technique; AUC, area under the ROC curve; Cl, 95\% confidence interval; Sens, sensitivity; Spec, specificity; PPV, positive predictive value; NPV, negative predictive value; $L R+$, positive likelihood ratio; LR-, negative likelihood ratio; P, significance level.

Additionally, ROC curve analysis showed that acetate N.I. was predictive of $\mathrm{PTB}<37$ weeks (area under the ROC curve, $\mathrm{AUC}=0.75,95 \% \mathrm{CI}=0.60-0.91),<32$ weeks $(\mathrm{AUC}=0.73$, $95 \% \mathrm{CI}=0.53-0.94)$, and delivery within 2 weeks of the index assessment (AUC $=0.77,95 \% \mathrm{CI}=0.58-0.96$ ) (Table 2 and Figure 3).

Normalized integrals of succinate, formate, lactate, glucose, glutamine/glutamate, alanine, and branched chain amino acids did not differ between term- and preterm-delivered women.

\section{Acetate Concentration Measured by Spectrophotometry}

Similar to our observation with acetate N.I., AceConc measured by the spectrophotometric technique was significantly higher in the preterm-delivered vs. term-delivered women $(0.63 \pm 0.04$ vs. $0.54 \pm 0.01 \mathrm{~g} / \mathrm{l} ; P=0.006)$ (Figure $2 \mathrm{~B}$ ), and correlated strongly with the ${ }^{1} \mathrm{H}-\mathrm{NMR}$ acetate N.I. $(r=0.69$, $P<0.00001$ ) (Figure 4).

Furthermore, acetate concentration measured by the spectrophotometric technique was predictive of $\mathrm{PTB}<37$ weeks $(\mathrm{AUC}=0.74,95 \% \mathrm{CI}=0.57-0.90)$ and delivery within 2 weeks of the index assessment $(\mathrm{AUC}=0.68,95 \% \mathrm{CI}=0.47-0.89)$, with an optimal cutoff value of $>0.53 \mathrm{~g} / \mathrm{l}$. However, its predictive performance for $\mathrm{PTB}<32$ weeks did not attain statistical significant in this limited sample (Table 2; Figure 3).

\section{CVF FFN, Ultrasound CL, and Vaginal pH}

The women destined to deliver preterm had significantly shorter mean CL $(21.1 \pm 4.8$ vs. $30.8 \pm 1.6 \mathrm{~mm})$ and greater than 12 -fold higher FFN concentration $(188 \pm 85$ vs. $15.2 \pm 3 \mathrm{ng} / \mathrm{ml})$ compared to their term counterparts (Table 1 and Figure S3 in Supplementary Material). Both FFN $(r=-0.7, P<0.00001)$ 
A $<37$ weeks gestation
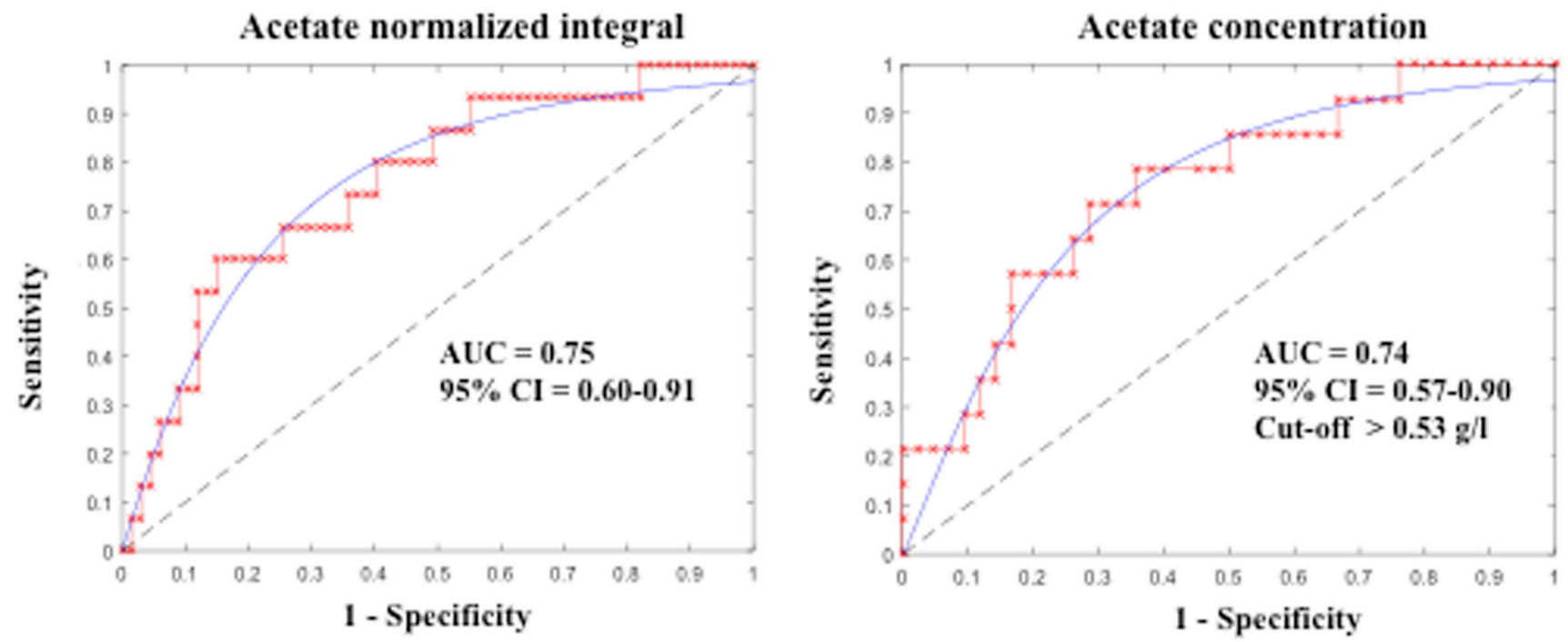

B

$\leq 2$ weeks of index assessment

Acetate normalized integral

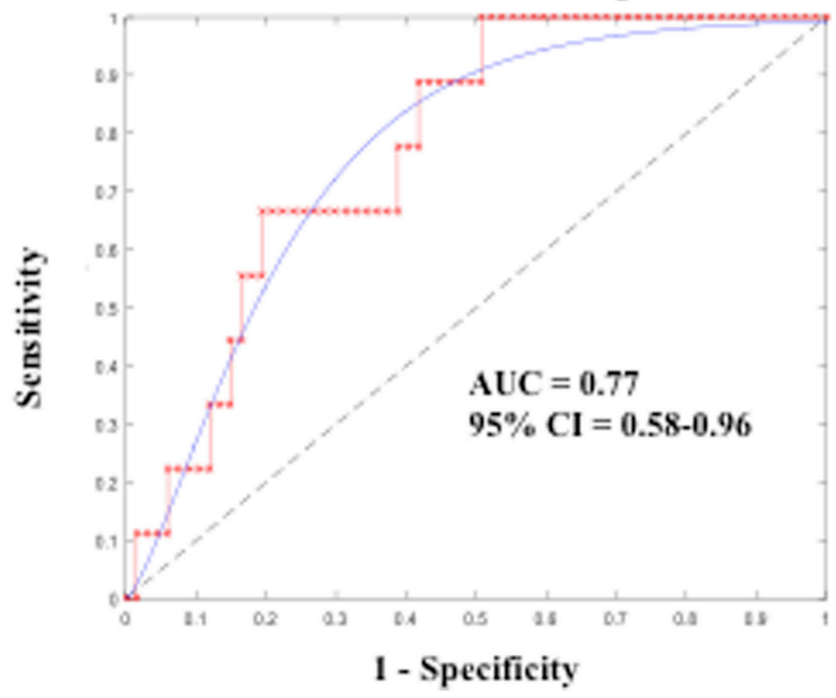

Acetate concentration

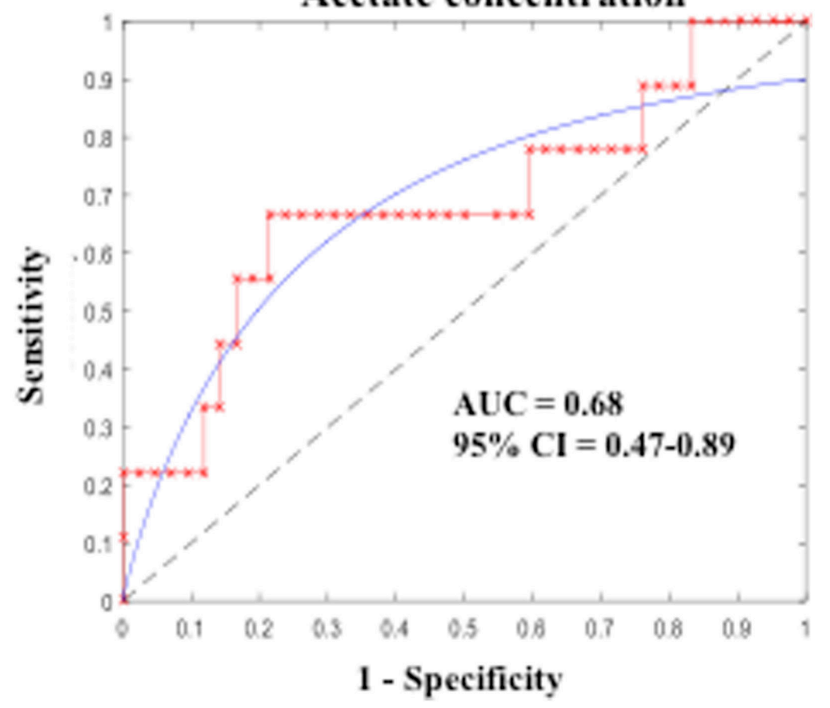

FIGURE 3 | Predictive accuracy of CVF acetate for preterm birth (A) before 37 weeks and (B) within 2 weeks of index assessment in pregnant women with symptoms of preterm labor. CVF acetate is measured by ${ }^{1} \mathrm{H}-\mathrm{NMR}$ (acetate normalized integral) and spectrophotometry (acetate concentration).

and CL $(r=0.4, P=0.01)$ correlated with gestational age at delivery (GAAD) (an indication of term or PTB) (Figure S4 in Supplementary Material).

Also, analysis of the area under the ROC curve showed FFN, and ultrasound CL were predictive of PTB $<37$ weeks (Table 3).

Vaginal $\mathrm{pH}$ did not differ between term- and pretermdelivered women (Table 1), but it correlated with acetate $(r=0.4$, $P=0.01)$ and lactate N.I.s $(r=-0.6, P<0.00001)$ (Figure S5 in Supplementary Material).

\section{Combined Predictive Performance of CVF Acetate, FFN, and Ultrasound CL}

Apart from the observation of comparable predictive capacities between CVF acetate, FFN, and ultrasound CL (Table 3), combining CVF acetate estimated by ${ }^{1} \mathrm{H}-\mathrm{NMR}$ and spectrophotometry with these widely used clinical assessment markers improved the prediction of PTB - the inclusion of either CVF acetate N.I. or acetate concentration with FFN and ultrasound CL in a binary logistic regression model improved prediction of PTB before 37 weeks gestation (AUC $=0.86$, sensitivity $83 \%$, specificity 
$89 \%, \mathrm{LR}+7.3$, and LR- 0.2 for acetate N.I. and $\mathrm{AUC}=0.80$, sensitivity $80 \%$, specificity $84 \%, L R+5.0$ and LR -0.2 for acetate concentration) than each of the three markers considered singly (Figures 5A,B). However, the combination of FFN and ultrasound $\mathrm{CL}$ alone indicated comparable predictive power to all three markers combined together $(\mathrm{AUC}=0.84)$ (Table 3$)$.

\section{DISCUSSION}

We have employed ${ }^{1} \mathrm{H}$-NMR spectroscopy to characterize the CVF metabolite profiles of a cohort of pregnant women

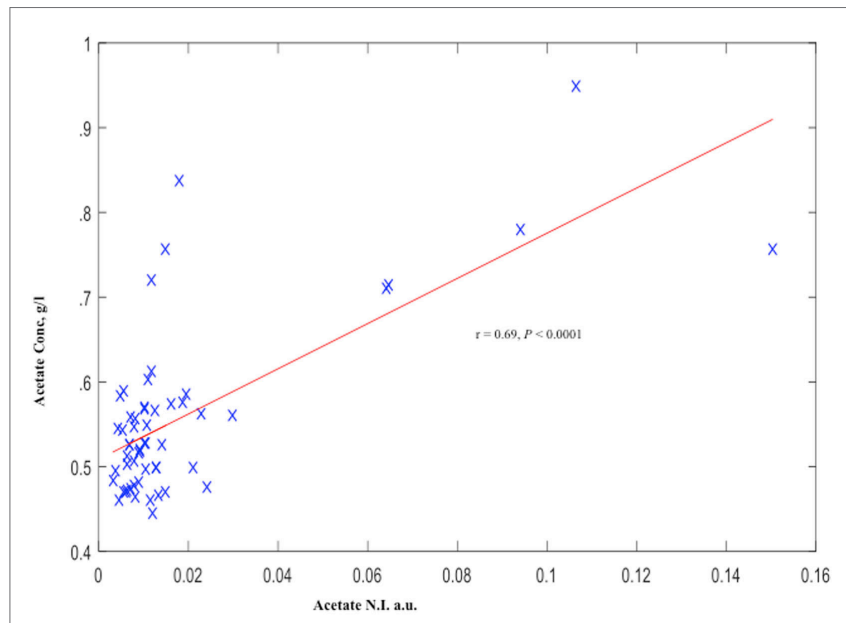

FIGURE 4 | Cervicovaginal fluid acetate of symptomatic pregnant women estimated by ${ }^{1} \mathrm{H}-\mathrm{NMR}$ and Spectrophotometry. a.u., arbitrary unit.

\section{A}

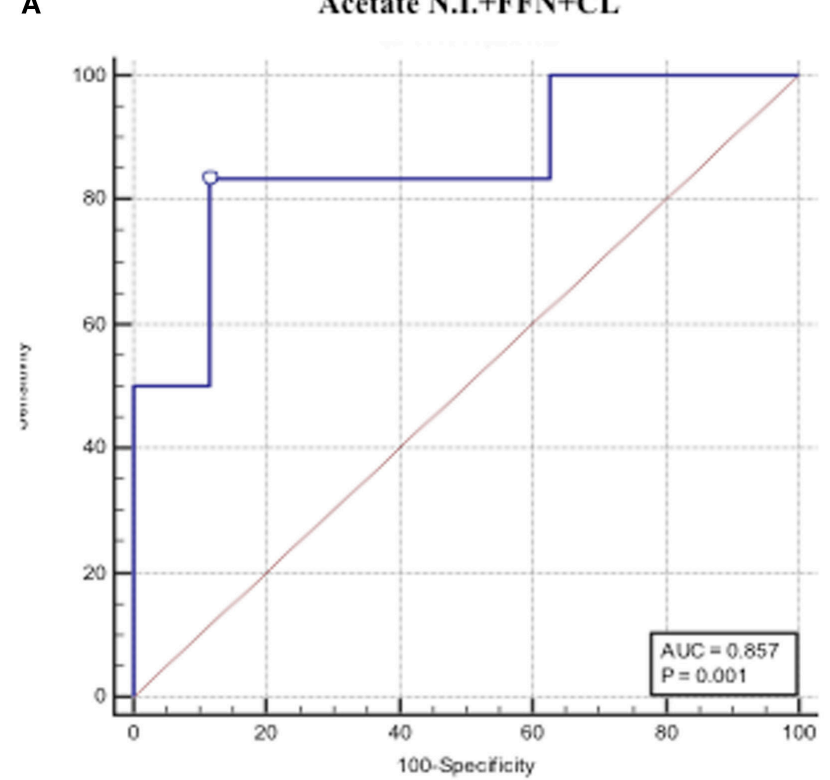

presenting with symptoms suggestive of threatened PTL at mid-gestation and determined their prognostic accuracy for PTB. Consistent with our previous report (18), we confirmed that CVF acetate appears predictive of PTB in this cohort. Furthermore, we have now validated this observation in this report employing commercially available enzyme-based spectrophotometric assay kits for acetic acid. We observed that CVF acetate predicted PTB as well as quantitative FFN and ultrasound $\mathrm{CL}$, and that when combined with these determinations, predictive accuracy for preterm delivery before 37 weeks gestation improved moderately. To our knowledge, this comparative analysis is the first of its kind.

Of the metabolites identified in the ${ }^{1} \mathrm{H}-\mathrm{NMR}$ spectra of CVF samples of women presenting with features of PTL, only acetate levels were associated with preterm delivery. The women destined to deliver prematurely had a twofold higher acetate N.I. compared to those who delivered at term. Acetate N.I. was also prognostic of PTB before 37 and 32 weeks of gestation, as well as of delivery within 2 weeks of the index assessment. Measurement of CVF acetate levels by ${ }^{1} \mathrm{H}-\mathrm{NMR}$, which is a marker of altered vaginal microbiota (6), is associated with imminent PTB.

However, acetate N.I. determination by ${ }^{1} \mathrm{H}-\mathrm{NMR}$ assessment does not lend itself to clinical use as an assay technique. We therefore validated ${ }^{1} \mathrm{H}-\mathrm{NMR}$ data by determining absolute concentrations of acetate in CVF by employing a commercial spectrophotometric assay technique from a randomly selected subset of the same symptomatic pregnant women. We determined that acetate concentration derived by spectrophotometry correlated strongly with ${ }^{1} \mathrm{H}-\mathrm{NMR}$-derived acetate N.I. being significantly higher in the women who ultimately delivered preterm. Additionally, acetate concentration was predictive
B

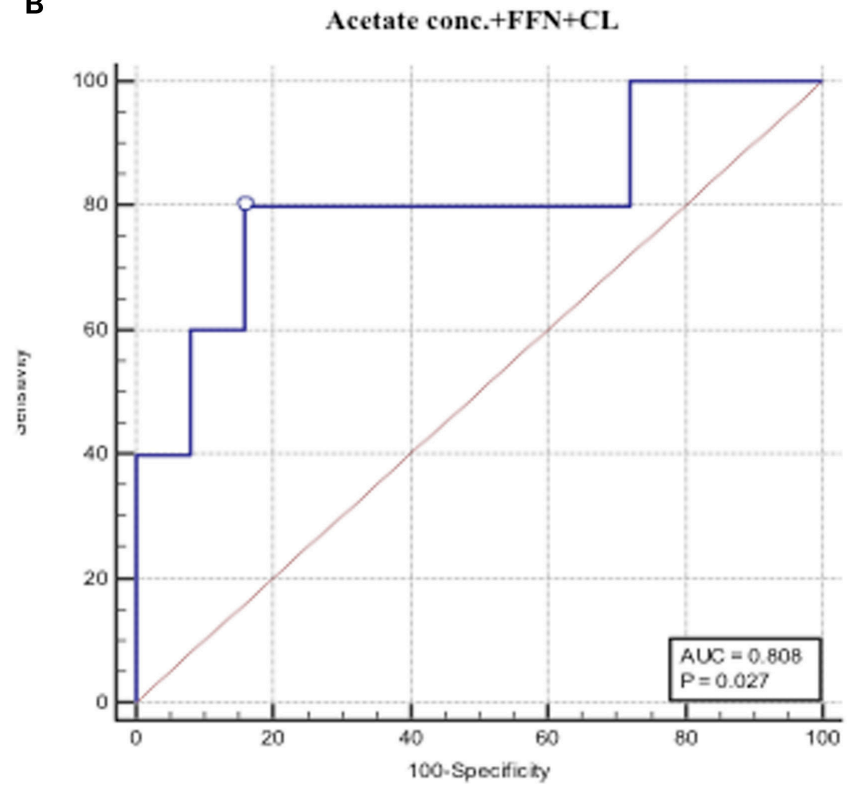

FIGURE 5 | AUROCs depicting combined PTB predictive performance of fetal fibronectin (FFN), cervical length and CVF acetate estimated by ${ }^{1} \mathrm{H}-\mathrm{NMR}(\mathrm{A})$, and spectrophotometry (B). 
TABLE 3 | Individual and combined predictive values of CVF acetate, fetal fibronectin, and cervical length for preterm birth.

\begin{tabular}{ll} 
Test & AUROC (95\% CI) \\
\hline Acetate N.I. & $0.75(0.60-91)$ \\
AceConc & $0.74(0.57-0.90)$ \\
Fetal fibronectin & $0.76(0.53-1.0)$ \\
Cervical length & $0.73(0.52-0.94)$ \\
Fetal fibronectin + cervical length & $0.84(0.64-1.0)$ \\
Fetal fibronectin + cervical length + acetate N.I. & $0.86(0.69-0.95)$ \\
Fetal fibronectin + cervical length + AceConc & $0.81(0.62-0.93)$
\end{tabular}

AUROC, area under the ROC curve; Acetate N.I., ${ }^{1} \mathrm{H}$-NMR acetate normalized integral; AceConc, acetate concentration measured by spectrophotometric technique.

of preterm delivery before 37 weeks gestation and of delivery within 2 weeks of the index assessment. However, predictive potential for delivery before 32 weeks did not attain statistical significance as only eight women in this subset delivered before 32 weeks gestation.

Conditions characterized by altered vaginal microflora such as intermediate flora, aerobic vaginitis, and BV have been implicated in infection/inflammation-induced preterm delivery. However, $\mathrm{BV}$ is the most investigated (3). The earlier the gestational age at the onset of PTL and possibly preterm premature rupture of membranes leading to PTB, the higher the likelihood of genital tract infection and inflammation (e.g., chorioamnionitis) (20). More than half of the women who delivered preterm in this study and approximately $10 \%$ of the entire study population delivered very prematurely before 32 weeks. In this category of PTBs, subclinical intrauterine infection is most often evident (20). As demonstrated in this study, identification of metabolic by-products (such as acetate) of pathogenic anaerobic bacteria seen in the above conditions could be used as a non-invasive, quick, and cost-effective proxy marker for the characterization of the prevailing vaginal microbial community and the attendant inflammatory state. Understanding these metabolite patterns in pregnant women may, therefore, aid understanding of the mechanisms of inflammation-induced preterm delivery, as well as uncover potential novel therapies.

We also demonstrated a relationship between vaginal $\mathrm{pH}$, acetate, and lactate N.I.s (Figure S5 in Supplementary Material), consistent with a potential metabolite signature pattern in women destined to deliver preterm. Vaginal $\mathrm{pH}$ increases with elevated acetate production by anaerobic bacteria (e.g., Gardnerella, Prevotella, Bacteroides, Mobilincus, Mycoplasma, etc.) characteristic of BV, while $\mathrm{pH}$ decreases with increase in lactate predominantly produced by Lactobacillus species characteristic of a healthy vaginal microbiota (6). These changes, together with increased recruitment of neutrophils, pro-inflammatory cytokines, and chemokines (e.g., IL-6 and IL-8), are also strongly associated with early third trimester rupture of fetal membranes $(8,21)$ often a precursor of idiopathic spontaneous PTB.

Although quantitative FFN and ultrasound CL are the most utilized clinical assessment markers for distinguishing women at risk of PTB in asymptomatic and symptomatic cohorts, there use is associated with a high rate of false positive results, low sensitivity, and positive predictive values for PTB (2). Combining both tests in midtrimester appears to improve their predictive accuracy of PTB (22). Furthermore, an association between genital tract infection/inflammation, short cervix, membrane activation and disruption, and leakage of FFN has been reported $(8,23)$. In this cohort, CVF acetate appeared as predictive of PTB as CL and FFN separately. Considering the limitations of FFN and ultrasound CL, we combined them with CVF acetate to determine whether the prognostication of PTB could be improved by the multiple biomarkers. We have observed that the combination yielded improved prediction of PTB.

In summary, we have reported that elevated CVF acetate (produced by mixed anaerobes) in women with symptoms of PTL appears predictive of preterm delivery, as well as delivery within 2 weeks of presentation, independently. This is supported by the established association of vaginal microbiota dominated by mixed anaerobic bacteria with the initiation of inflammation-associated PTL and birth. In pregnant women presenting with symptoms suggestive of PTL, a clinical assay of acetate in CVF may prove of clinical utility for predicting PTB singly and in combination with quantitative FFN and ultrasound CL, if our observations are confirmed in larger studies. Whether CVF acetate has clinical utility for predicting PTB in asymptomatic pregnant women is yet to be determined.

\section{AUTHOR CONTRIBUTIONS}

EA conducted the laboratory experiments, analyzed and interpreted the data, and drafted the manuscript. SR supervised the NMR experiments and contributed to data analysis and the manuscript. VS recruited study participants, obtained tissue samples, carried out the clinical studies, and contributed to data analysis and the manuscript. GS contributed to the study concept, project supervision, and the manuscript. MP supervised NMR experiments and contributed to the manuscript. DA conceived the studies, supervised the project, contributed to the analysis and interpretation of data, and writing the manuscript. All authors approved the final draft for publication. Parts of this work have been presented at the 63rd Annual Scientific Meeting of the Society for Reproductive Investigation, Montreal 2016, O-090 (Oral); the 18th annual conference of the British Maternal and Fetal Medicine Society, Birmingham 2016, PP.16 (Poster); and the 24th annual meeting and exhibition of the International Society of Magnetic Resonance in Medicine, Singapore 2016, 2414 (Poster).

\section{ACKNOWLEDGMENTS}

We are grateful to the women who consented to participate in these studies and to the service user involvement panels who provided useful advice during the planning and execution of these experiments.

\section{FUNDING}

This study was funded in part by the Medical Research Council, UK (Grant number: MR/J014788/1); and EA was supported with a Ph.D. studentship from the Niger Delta Development Commission and Bayelsa State Scholarship Board of Nigeria. 


\section{SUPPLEMENTARY MATERIAL}

The Supplementary Material for this article can be found online at http://journal.frontiersin.org/article/10.3389/fmed.2016.00048

\section{Acetic Acid Spectrophotometric Assay}

The assay principle is based on the determination of acetate concentration by the generation of $\mathrm{NADH}$ from NAD+, which is then quantified by the increase in absorbance at $340 \mathrm{~nm}$ (24). The enzymes involved in the four-step reaction and supplied with the assay kit include: (1) acetate kinase, (2) phosphotransacetylase, (3) ADP-glucokinase (ADP-GK), and (4) glucose-6-phosphate dehydrogenase (G6P-DH). Acetate is converted through the following reaction, which is positively related to the eventual formation of $\mathrm{NADH}$ :

1. Acetic acid + ATP $\stackrel{\text { Acetate kinase }}{\longrightarrow}$ acetyl-phosphate + ADP

2. Acetyl-phosphate $+\mathrm{CoA} \stackrel{\text { Phosphotransacetylase }}{\longrightarrow}$ acetyl$\mathrm{CoA}+\mathrm{P}_{\mathrm{i}}$

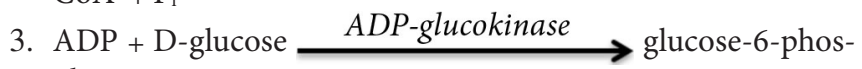
phate + AMP

4. Glucose-6-phosphate $+\mathrm{NAD}^{+} \stackrel{\text { G6P-dehydrogenase }}{\longrightarrow}$ 6-phosphogluconate $+\mathrm{NADH}+\mathrm{H}^{+}$

The reaction is a positive reaction indicated by the increase in absorbance. The acetic acid GK assay kit is an endpoint type

\section{REFERENCES}

1. Blencowe H, Cousens S, Oestergaard MZ, Chou D, Moller A-B, Narwal R, et al. National, regional, and worldwide estimates of preterm birth rates in the year 2010 with time trends since 1990 for selected countries: a systematic analysis and implications. Lancet (2012) 379(9832):2162-72. doi:10.1016/ S0140-6736(12)60820-4

2. Heng YJ, Liong S, Permezel M, Rice GE, Di Quinzio MK, Georgiou HM. Human cervicovaginal fluid biomarkers to predict term and preterm labor. Front Physiol (2015) 6:151. doi:10.3389/fphys.2015.00151

3. Witkin $\mathrm{S}$. The vaginal microbiome, vaginal anti-microbial defence mechanisms and the clinical challenge of reducing infection-related preterm birth. BJOG (2015) 122(2):213-8. doi:10.1111/1471-0528.13115

4. Al-Mushrif S, Eley A, Jones B. Inhibition of chemotaxis by organic acids from anaerobes may prevent a purulent response in bacterial vaginosis. J Med Microbiol (2000) 49(11):1023-30. doi:10.1099/0022-1317-49-11-1023

5. Mirmonsef P, Zariffard MR, Gilbert D, Makinde H, Landay AL, Spear GT. Short-chain fatty acids induce pro-inflammatory cytokine production alone and in combination with toll-like receptor ligands. Am J Reprod Immunol (2012) 67(5):391-400. doi:10.1111/j.1600-0897.2011.01089.x

6. Aldunate M, Srbinovski D, Hearps AC, Latham CF, Ramsland PA, Gugasyan R, et al. Antimicrobial and immune modulatory effects of lactic acid and short chain fatty acids produced by vaginal microbiota associated with eubiosis and bacterial vaginosis. Front Physiol (2015) 6:164. doi:10.3389/fphys. 2015.00164

7. Pararas MV, Skevaki CL, Kafetzis DA. Preterm birth due to maternal infection: causative pathogens and modes of prevention. Eur J Clin Microbiol Infect Dis (2006) 25(9):562-9. doi:10.1007/s10096-006-0190-3

8. Agrawal V, Hirsch E. Intrauterine infection and preterm labor. Semin Fetal Neonatal Med (2012) 17(1):12-9. doi:10.1016/j.siny.2011.09.001

9. Vrachnis N, Karavolos S, Iliodromiti Z, Sifakis S, Siristatidis C, Mastorakos G, et al. Impact of mediators present in amniotic fluid on preterm labour. In Vivo (2012) 26(5):799-812. assay specific for acetate with its lowest sensitivity limit $\sim 0.1 \mathrm{~g} / \mathrm{l}$ $\left(R^{2}=0.9997\right)$. A calibration curve indicating the linearity of K-ACETGK 08/14 was produced by plotting absorbance at $340 \mathrm{~nm}$ against concentrations of the acetic acid standards (gram per liter) $\left(R^{2}=0.975\right.$, detection limit: $\left.1.8 \mathrm{~g} / \mathrm{l}\right)$ (Figure S2 in Supplementary Material). Different concentrations (calibrators) of the acetic acid standard (1.8 g/l) were made be serial dilution (i.e., 1.8, 1.2, 0.9, $0.6,0.45,0.36,0.3,0.225,0.2$, and $0.15 \mathrm{~g} / \mathrm{l})$. From the calibration curve, concentrations of acetate in the samples were ascertained after measurement of their absorbance at $340 \mathrm{~nm}$.

FIGURE S1 | 2-D ${ }^{1} \mathrm{H}-{ }^{13} \mathrm{C}$ HSQC NMR spectrum of cervicovaginal fluid showing confirmed metabolites at $294 \mathrm{~K}$. BCAA, branched chain amino acids (leucine, isoleucine, and valine); HSQC, heteronuclear single quantum correlation spectroscopy.

FIGURE S2 | Calibration curve indicating the linearity of K-ACETGK. The reactions from which this calibration curve was generated were performed at $37^{\circ} \mathrm{C}$ for about 5 min using a TECAN Infinite M200 microplate reader (detection limit $=1.8 \mathrm{~g} / \mathrm{l}$ or $0.03 \mathrm{~mol} / \mathrm{l})$.

FIGURE S3 | Clinical assessment methods for preterm birth (A) quantitative fetal fibronectin level and (B) ultrasound cervical length in relation to delivery outcomes. Data are presented as mean \pm SE. ${ }^{*} P$ value $<0.05$

FIGURE S4 | Association of quantitative fetal fibronectin (FFN) level (A), ultrasound cervical length (B), and gestational age at delivery (GAAD).

FIGURE S5 | Association of vaginal pH and CVF acetate (A) and lactate (B) normalized integrals in symptomatic pregnant women. a.u., arbitrary unit.

10. Witkin SS, Mendes-Soares H, Linhares IM, Jayaram A, Ledger WJ, Forney LJ. Influence of vaginal bacteria and D-and L-lactic acid isomers on vaginal extracellular matrix metalloproteinase inducer: implications for protection against upper genital tract infections. MBio (2013) 4(4):e460-413. doi:10.1128/mBio.00460-13

11. Laghi L, Picone G, Cruciani F, Brigidi P, Calanni F, Donders G, et al. Rifaximin modulates the vaginal microbiome and metabolome in women affected by bacterial vaginosis. Antimicrob Agents Chemother (2014) 58(6):3411-20. doi:10.1128/AAC.02469-14

12. Srinivasan S, Morgan MT, Fiedler TL, Djukovic D, Hoffman NG, Raftery D, et al. Metabolic signatures of bacterial vaginosis. MBio (2015) 6(2):e204-15. doi:10.1128/mBio.00204-15

13. Vitali B, Cruciani F, Picone G, Parolin C, Donders G, Laghi L. Vaginal microbiome and metabolome highlight specific signatures of bacterial vaginosis. Eur J Clin Microbiol Infect Dis (2015) 34(12):2367-76. doi:10.1007/ s10096-015-2490-y

14. Chaudry AN, Travers PJ, Yuenger J, Colletta L, Evans P, Zenilman JM, et al. Analysis of vaginal acetic acid in patients undergoing treatment for bacterial vaginosis. JClin Microbiol (2004) 42(11):5170-5. doi:10.1128/ JCM.42.11.5170-5175.2004

15. Rahkonen L, Rutanen EM, Unkila-Kallio L, Nuutila M, Nieminen P, Sorsa T, et al. Factors affecting matrix metalloproteinase- 8 levels in the vaginal and cervical fluids in the first and second trimester of pregnancy. Hum Reprod (2009) 24(11):2693-702. doi:10.1093/humrep/dep284

16. Donders G. Reducing infection-related preterm birth. BJOG (2015) 122(2):219. doi:10.1111/1471-0528.13109

17. Perunovic ND, Rakic MM, Nikolic LI, Jankovic SM, Aleksic ZM, Plecas DV, et al. The association between periodontal inflammation and labor triggers (elevated cytokine levels) in preterm birth: a cross-sectional study. JPeriodontol (2015) 87(3):248-56. doi:10.1902/jop.2015. 150364

18. Amabebe E, Reynolds S, Stern VL, Parker JL, Stafford GP, Paley MN, et al. Identifying metabolite markers for preterm birth in cervicovaginal fluid 
by magnetic resonance spectroscopy. Metabolomics (2016) 12(4):1-11. doi:10.1007/s11306-016-0985-x

19. Madhu B, Narita M, Jauhiainen A, Menon S, Stubbs M, Tavaré S, et al. Metabolomic changes during cellular transformation monitored by metabolite-metabolite correlation analysis and correlated with gene expression. Metabolomics (2015) 11(6):1848-63. doi:10.1007/s11306-0150838-z

20. Goldenberg RL, Culhane JF, Iams JD, Romero R. Preterm birth 1 - epidemiology and causes of preterm birth. Lancet (2008) 371(9606):75-84. doi:10.1016/ S0140-6736(08)60074-4

21. Simhan HN, Caritis SN, Krohn MA, Hillier SL. The vaginal inflammatory milieu and the risk of early premature preterm rupture of membranes. Am J Obstet Gynecol (2005) 192(1):213-8. doi:10.1016/j.ajog.2004.07.021

22. DeFranco EA, Lewis DF, Odibo AO. Improving the screening accuracy for preterm labor: is the combination of fetal fibronectin and cervical length in symptomatic patients a useful predictor of preterm birth? A systematic review. Am J Obstet Gynecol (2013) 208(3):.e1-6. doi:10.1016/j.ajog.2012.12.015

23. Holst RM, Jacobsson B, Hagberg H, Wennerholm UB. Cervical length in women in preterm labor with intact membranes: relationship to intra-amniotic inflammation/microbial invasion, cervical inflammation and preterm delivery. Ultrasound Obstet Gynecol (2006) 28(6):768-74. doi:10.1002/ uog. 3837

24. Davies PG, Venkatesh B, Morgan TJ, Presneill JJ, Kruger PS, Thomas BJ, et al. Plasma acetate, gluconate and interleukin- 6 profiles during and after cardiopulmonary bypass: a comparison of plasma-lyte 148 with a bicarbonatebalanced solution. Crit Care (2011) 15(1):R21. doi:10.1186/cc9966

Conflict of Interest Statement: The authors declare that this research was conducted in the absence of any commercial or financial relationships that could be construed as a potential conflict of interest.

Copyright (c) 2016 Amabebe, Reynolds, Stern, Stafford, Paley and Anumba. This is an open-access article distributed under the terms of the Creative Commons Attribution License (CC BY). The use, distribution or reproduction in other forums is permitted, provided the original author(s) or licensor are credited and that the original publication in this journal is cited, in accordance with accepted academic practice. No use, distribution or reproduction is permitted which does not comply with these terms. 